\title{
Diversity Combining and Piezoelectric Beam Steering for Multi-Element VLC Networks
}

\author{
Yusuf Said Eroglu ${ }^{\dagger}$, Ismail Guvenc ${ }^{\dagger}$, Alphan Sahin ${ }^{\dagger}$, Nezih Pala ${ }^{\dagger}$ and Murat Yuksel ${ }^{\ddagger}$ \\ ${ }^{\dagger}$ Department of Electrical and Computer Engineering, Florida International University, Miami, FL \\ ‡Department of Computer Science and Engineering, University of Nevada, Reno, NV \\ Email: \{yerog001,iguvenc,asahin,npala\}@fiu.edu, yuksem@cse.unr.edu
}

\begin{abstract}
Visible light communications (VLC) is an emerging wireless communication technology that can serve high speed data rates by using existing lighting infrastructure without congesting the radio spectrum. This paper studies the effect of various multi-element VLC transmitter and receiver configurations on the link quality. With this purpose, the signal-tointerference-plus-noise-ratio (SINR) distribution is investigated via extensive simulations considering a multipath environment with different transceiver configurations and diversity combining techniques. Additionally, we propose a beam steering technique using the piezo-electric based tilting of LEDs to improve the SINR, which tilts the LEDs with piezoelectric actuators towards the location of the receiver. Simulation results show that significant gains can be obtained on SINR performance by using optimized transceiver configurations and diversity combining at the receiver. Moreover, even with small titling angles at the transmitter, up to 12 $\mathrm{dB}$ SINR gain is obtained using beam steering.
\end{abstract}

\section{Keywords}

Angle diversity, free space optics (FSO), Li-Fi, optical wireless communication (OWC), piezoelectric actuators, optimal combining (OPC).

\section{INTRODUCTION}

Visible light communications (VLC) is a complementary technology to radio frequency ( $R F$ ) communications, which can potentially improve the data throughput for next generation wireless networks while simultaneously providing illumination. With VLC technology, light sources, particularly LEDs, can be used to transmit digital data by modulating the intensity of the light. A major advantage of VLC is that the performance of VLC systems is not affected by RF interference as the frequency of the visible light is significantly

This work is supported in part by NSF CNS awards 1422354 and 1422062, ARO DURIP award W911NF-14-1-0531, and NASA NV Space Consortium.

Permission to make digital or hard copies of all or part of this work for personal or classroom use is granted without fee provided that copies are not made or distributed for profit or commercial advantage and that copies bear this notice and the full citation on the first page. Copyrights for components of this work owned by others than ACM must be honored. Abstracting with credit is permitted. To copy otherwise, or republish, to post on servers or to redistribute to lists, requires prior specific permission and/or a fee. Request permissions from permissions@ acm.org.

VLCS'16, October 03-07, 2016, New York City, NY, USA

(c) 2016 ACM. ISBN 978-1-4503-4253-7/16/10 . \$ $\$ 15.00$

DOI: http://dx.doi.org/10.1145/2981548.2981557 higher than the RF. Therefore, it provides additional wireless capacity using a very large visible light spectrum, without impacting ongoing RF communications. Other advantages of VLC networks include: 1) secure communications by isolation, since visible light does not penetrate through walls; 2) energy efficiency, since optoelectronic transmitters use significantly lower source power per bit, and existing solid-state lighting infrastructure will be used for communication; and 3) high-accuracy localization by using directionality of VLC transmissions [1-3].

In the literature, transmitter and receiver architectures with multiple LEDs are proposed for improving the link quality of VLC networks. In [4], it is claimed that installing single LEDs with short distance increases the installation cost, since all the transmitters are needed to be individually installed on the ceilings and connected to electricity and network. Alternatively, a multi-element angle-diversity transmitter, where multiple LEDs are placed to same access point (AP), is proposed in order to reduce the cable and circuitry costs while providing ease of installation and maintenance. As a disadvantage, it is shown that these transmitters yield significantly lower signal-to-interferenceplus-noise-ratio (SINR) than single-element VLC transmitters. In [5], an LED partitioning algorithm is proposed to increase the SINR of multi-element transmitters. In $[2,6]$, it is demonstrated that multi-element transmitters provide highly accurate direction estimation as they provide better angular resolution with highly directive LEDs.

When multiple photo detectors (PDs) are employed at the receiver, the link quality can be significantly improved with diversity combining techniques [7-10]. For example, in [10], four different receiver combining schemes are studied, and it is shown that link quality performance can be enhanced by capturing the correlations of the received signal strengths at different PDs. However, to the best of our knowledge, there has not been a study that investigates the performance of multi-element receivers and combining techniques with angle diversity transmitters. Another way of improving the signal quality is the beam steering and beam tracking. In [11], an inexpensive solution, which utilizes piezoelectric actuators to tilt the LEDs, is proposed for steering the beams. Again, to the best of our knowledge, there has been no study that investigates the achievable gains with beam steering for VLC networks.

In this paper, we consider the deployment of a fixed number of LEDs within a room, using 1) a single-LED transmitter architecture, and 2) a 7-LED multi-element angle diversity transmitter architecture. We investigate their per- 
formances under fair conditions, and with different receiver structures. We propose multi-element receivers as a solution to the low SINR problem of multi-element transmitters, and investigate optimum parameters and combining schemes for closing the "SINR gap" with the single-LED transmitter architecture. At VLC receiver, we consider four different diversity combining techniques: equal gain combining (EGC), selective best combining (SBC), maximal ratio combining (MRC), and optimal combining (OPC). Subsequently, using a rigorous VLC simulation platform, we evaluate SINR performance by capturing its cumulative distribution function (CDF) across the room that VLC transmitters are deployed. Our results show that with usage of angle diversity transmitter, performance gains due to use of OPC increases, while a larger field of view (FOV) and tilt angle between PDs becomes necessary at the receiver.

Additionally, we present a novel piezoelectric beam steering scheme to further improve the received signal strength at a mobile VLC receiver. In particular, the connected LED tracks the user location and changes beam direction at minor tilt angles with the help of piezoelectric actuators to provide higher signal power. Simulations results show that dynamic beam steering of LEDs on the order of $5^{\circ}$ can introduce extensive SINR gains, especially on regions with low SINR.

The rest of the paper is organized as follows. In Section II, VLC propagation channel model is explained and multi-element transceiver architectures are introduced. In Section III, multi-element combining schemes at the receiver are introduced. Piezoelectric beam steering method is explained in Section IV, and simulation results are presented in Section V. Finally, the last section concludes the paper.

\section{SYSTEM MODEL}

In this section, line-of-sight (LOS) and non-line-of-sight (NLOS) components of the channel is explained, and multielement transmitter and receiver structures are presented.

\subsection{Channel Model}

A light source $\mathcal{S}$ with a unit transmit power can be represented with three parameters as $\mathcal{S}=\left\{\mathbf{r}_{\mathrm{src}}, \mathbf{n}_{\mathrm{src}}, n\right\}$, where $\mathbf{r}_{\mathrm{src}}$ is the location of the light source, $\mathbf{n}_{\mathrm{src}}$ is the direction of light source, and $n$ is the LED mode, which characterizes the directivity of the LED. A receiver $\mathcal{R}$ can be modeled as $\mathcal{R}=\left\{\mathbf{r}_{\text {rec }}, \mathbf{n}_{\text {rec }}, A_{\mathrm{R}}, \theta_{\mathrm{FOV}}\right\}$, where $\mathbf{r}_{\text {rec }} \in \mathbb{R}^{3 \times 1}$ is the location of the $\mathrm{PD}, \mathbf{n}_{\mathrm{rec}} \in \mathbb{R}^{3 \times 1}$ is the direction of the $\mathrm{PD}, A_{\mathrm{R}}$ is the area in $\mathrm{m}^{2}$, and $\theta_{\text {FOV }}$ is the FOV of the PD.

The LOS component of the channel between source $\mathcal{S}$ and receiver $\mathcal{R}$ is given by [12]

$$
h^{0}(\mathcal{S}, \mathcal{R})=\frac{n+1}{2 \pi} \cos ^{n}(\phi) \cos (\theta) \frac{A_{\mathrm{R}}}{\mathrm{R}^{2}} \Pi\left(\frac{\theta}{\theta_{\mathrm{FOV}}}\right),
$$

where $\phi$ is the angle between the source direction vector and the incidence vector, $\theta$ is the angle between the PD direction vector and incidence vector, $R$ is the distance between the source and the receiver, and $\Pi(\cdot)$ is the rectangular function (1 for $|x| \leq 1$ and 0 for $|x|>1$ ).

The NLOS component of the channel is obtained based on multiple bounce impulse response model [12]. As the light can reach the PD after several reflections, the channel impulse response due to the NLOS components can be written a)

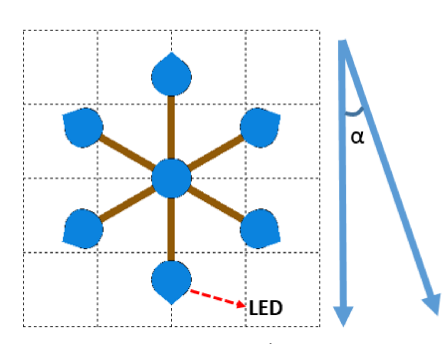

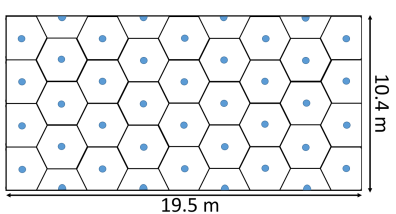

b)

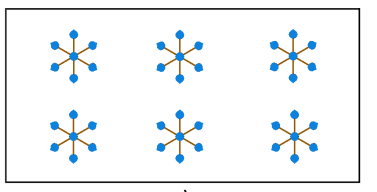

c)
Figure 1: Transmitter structures and deployment strategies. Fig. 1(a) shows the top view of 7-LED transmitter, and angle $\alpha$ between center and side LEDs. Fig. 1(b) and Fig. 1(c) show the layouts for 40 single-LED transmitters and six 7-LED transmitters in the room, respectively.

as

$$
h(\mathcal{S}, \mathcal{R})=\sum_{d=0}^{\infty} h^{(d)}(\mathcal{S}, \mathcal{R})
$$

where $d$ is the reflection order. Theoretically, $h^{(d)}(\mathcal{S}, \mathcal{R})$ can be expressed as a recursive function [12], given by

$$
\begin{array}{r}
h^{(d)}(\mathcal{S}, \mathcal{R})=\int_{S} \rho_{\text {refl }} \\
\times h^{0}\left(\mathcal{S},\left\{\mathbf{r}_{\text {reff }}, \mathbf{n}_{\text {reff }}, \mathrm{d} A, \frac{\pi}{2}\right\}\right) \\
* h^{(d-1)}\left(\left\{\mathbf{r}_{\text {reff }}, \mathbf{n}_{\text {refl }}, 1\right\}, \mathcal{R}\right) .
\end{array}
$$

In $(3), *$ is the convolution operation, $\mathbf{r}_{\text {refl }}$ and $\mathbf{n}_{\text {refl }}$ stands for the location and the direction of the reflector, respectively, $\mathrm{d} A$ is the infinitesimal area of the reflector, and $\rho_{\text {refl }} \in$ $[0,1)$ is the reflection coefficient. In addition, the FOV and the mode $n$ of the reflector, such as the walls in the room, are set to $\pi / 2$ and 1 , respectively.

\subsection{Multi-Element Transmitter Architectures}

Two different transmitter architectures and deployment strategies are shown in Fig. 1. For the first configuration, we assume that there is a single LED per transmitter, which is steered toward the floor. For the second configuration, there are 7 LEDs for each VLC transmitter, where one of the LEDs is steered towards the floor and the rest are tilted by an angle of $\alpha$ expressed in degrees. They are also separated from each other with $60^{\circ}$ azimuth angle. Throughout the paper, we assume that each LED serves to a different user, even though they are on the same transmitter. Therefore, there is only one LED from which the receiver receives the signal and we call it as the desired LED. All the other LEDs cause interference signals to the desired LED transmission. In [4], we studied two more multi-LED structures but with no consideration of diversity combining at the receiver. In this paper, we focus on the gains attainable by this feature, and the results are generalizable to other multi-LED transmitters.

\subsection{Multi-Element Receiver Architectures}

In the first configuration, the receiver has only a single PD. For the second configuration, there are three PDs, each with a tilt angle $\beta$ expressed in degrees from the normal of the receiver. In the third configuration, we consider a receiver structure with seven PDs. While the direction of 
one of the PDs is parallel to the direction of the receiver, a second layer of PDs are tilted with the angle $\beta$ in degrees. We assume that the FOV $\beta_{\mathrm{FOV}}$ and the surface area $A_{R}$ of PDs are identical.

\section{COMBINING TECHNIQUES}

In this section, different combining techniques, i.e., EGC, $\mathrm{SBC}, \mathrm{MRC}$, and OPC, are investigated for the aforementioned receiver and transmitter configurations and corresponding SINR calculations are discussed.

\subsection{Single-Element Receiver}

With a single $\mathrm{PD}$, the receive diversity techniques cannot be implemented as the receiver observes only one copy of the transmitted signal. In this case, the SINR is calculated as:

$$
S I N R=\frac{P_{\ell_{\mathrm{d}}}^{2}}{N_{0} B+\sum_{\ell=1, \ell \neq \ell_{\mathrm{d}}}^{N_{\mathrm{L}}} P_{\ell}^{2}},
$$

where $P_{\ell}$ is the received signal strength from the $l$ th LED, $\ell_{\mathrm{d}}$ stands for desired LED, $N_{\mathrm{L}}$ stands for the number of LEDs, $\sum_{\ell=1, \ell \neq \ell_{\mathrm{d}}}^{N_{\mathrm{L}}} P_{\ell}^{2}$ represents the interference signal power from interfering LEDs, $N_{0}$ is the spectral density of the additive white Gaussian noise (AWGN), and $B$ is the communication bandwidth.

\subsection{Multi-Element Receiver}

Multiple PDs can be used to improve the SINR over single LED scenario as:

$$
S I N R=\frac{\left(\sum_{p=1}^{N_{\mathrm{P}}} w_{p} P_{p, \ell_{\mathrm{d}}}\right)^{2}}{\sum_{p=1}^{N_{\mathrm{P}}} w_{p}^{2} N_{0} B+\sum_{\ell=1, \ell \neq \ell_{\mathrm{d}}}^{N_{\mathrm{L}}}\left(\sum_{p=1}^{N_{\mathrm{P}}} w_{p} P_{p, \ell}\right)^{2}},
$$

where $w_{p}$ is the weight for $p$ th $\mathrm{PD}$, and the second part in the denominator represents the sum of all interference signal powers at all PDs from all LEDs excluding the desired LED. The weights for the different diversity combining techniques are given as follows:

- Equal Gain Combining: In EGC, all weights $w_{p}$ in (5) are identical (equal to 1), and the receive combining does not yield an extra benefit over single PD detector. In contrast, EGC can cause worse combining SINR performance. This is because the received signal may be heavily directional in certain cases, and therefore some of the PDs may have very low SINRs.

- Selective Best Combining: For the SBC, the SINR is calculated in a similar way as the single $\mathrm{PD}$ receiver case in (4). In particular, the PD giving the highest SINR is selected, and the signal from other PDs are not used. Therefore, the weight in (5) for the PD with best SINR is 1 , while the rest of the weights are set to 0 . The SBC yields a high SINR gain in comparison to single PD case and EGC. Since a single $\mathrm{PD}$ receives the light from a narrower angle, it receives less interference.

- Maximum Ratio Combining: In MRC, each PD is

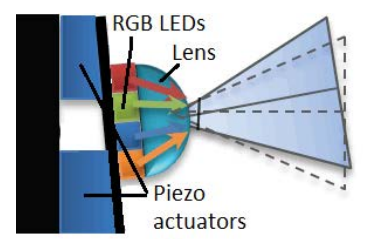

(a)

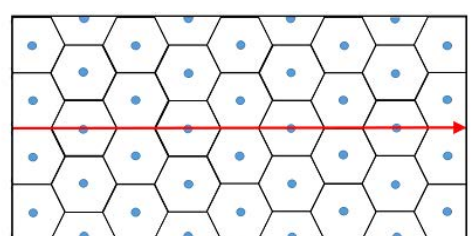

(b)
Figure 2: (a) Beam steering using piezoelectric actuators. (b) The trajectory used for beam steering simulation in Section V.

weighted proportionally with their SINR as:

$$
w_{p}=\frac{P_{p, \ell_{\mathrm{d}}}^{2}}{N_{0} B+\sum_{\ell=1, \ell \neq \ell_{\mathrm{d}}}^{N_{\mathrm{L}}} P_{p, \ell}^{2}} .
$$

- Optimal Combining: In OPC, the correlations between the signals observed at different PDs are exploited. When the dominant part of the channel is LOS (e.g., the single LED scenario in Fig. 1(b)), OPC gives only marginal gains when compared with the MRC. On the other hand, with multi-element VLC scenario, since LEDs are directed towards different angles, reflected signals weights an important amount in total received signal power, and OPC may significantly outperform MRC. The weights of optimal combining can be calculated as $\mathbf{w}_{p}=\mathbf{R}_{\mathrm{n}}^{-1} \mathbf{u}_{\ell_{\mathrm{d}}}$, where $\mathbf{u}_{\ell_{\mathrm{d}}}=\left[P_{1, \ell_{\mathrm{d}}}, P_{2, \ell_{\mathrm{d}}}, \ldots, P_{p, \ell_{\mathrm{d}}}\right]^{T}$ is the desired received signal, $\mathbf{R}_{\mathrm{n}}$ is the interference-plus-noise correlation matrix of the received signal, given by [13]

$$
\mathbf{R}_{\mathrm{n}}=N_{0} B \mathbf{I}+\sum_{\ell=1, \ell \neq \ell_{\mathrm{d}}}^{N_{\mathrm{L}}} E\left[\mathbf{u}_{\ell} \mathbf{u}_{\ell}^{T}\right],
$$

where $\mathbf{I}$ is identity matrix, and $\mathbf{u}_{\ell}=\left[P_{1, \ell}, P_{2, \ell}, \ldots, P_{P, \ell}\right]$ is the interference signal power from $\ell$ th LED.

\section{PIEZOELECTRIC BEAM STEERING}

VLC networks can provide highly accurate localization information [1-3], and this location information can be used for beam steering to further enhance the communications performance. Beam steering is commonly used in free space optic (FSO) systems. Since FSO light beams are highly directional, an accurate steering is a necessity for these systems. In contrary, VLC light beams have relatively broader divergence angles, and diffusion property of lights are used for both illumination and communication purposes. However, by manipulating the orientation of the light source, a gain in the SINR is possible, especially when more directional LEDs (i.e., with higher LED mode) are used.

An inexpensive method for beam steering is using piezoelectric actuators in order to change the direction of LEDs. Piezoelectric actuators convert electrical energy to mechanical energy and allows precisely controllable physical size change. They are used to adjust lenses, mirrors, or other equipment [11]. In VLC case, piezoelectric actuators can be used to change direction of LEDs as in Fig. 2(a).

In this paper, we consider that the LEDs will be steered to track the location of the receiver, which is assumed to be known by the transmitter. Since each LED serves a small area, the required steering angle is also small, which makes piezoelectric actuators a great fit for this task. When a user arrives at a location where further steering of an LED 
Table 1: Simulation parameters.

\begin{tabular}{|c|c|}
\hline LED mode, $n$ & 7.0459 \\
\hline Transmission power of an LED, $P_{\mathrm{t}}$ & $3 \mathrm{~W}$ \\
\hline Modulation bandwidth, $B$ & $20 \mathrm{MHz}$ \\
\hline AWGN spectral density, $N_{0}$ & $2.5 \times 10^{-23} \mathrm{~A} / \mathrm{Hz}$ \\
\hline Effective surface area of PDs, $A_{\mathrm{R}}$ & $10 \mathrm{~mm}^{2}$ \\
\hline Reflection coefficient (walls), $\rho_{\text {refl }}$ & 0.8 \\
\hline Reflection coefficient (floor, ceiling), $\rho_{\text {refl }}$ & 0.3 \\
\hline
\end{tabular}

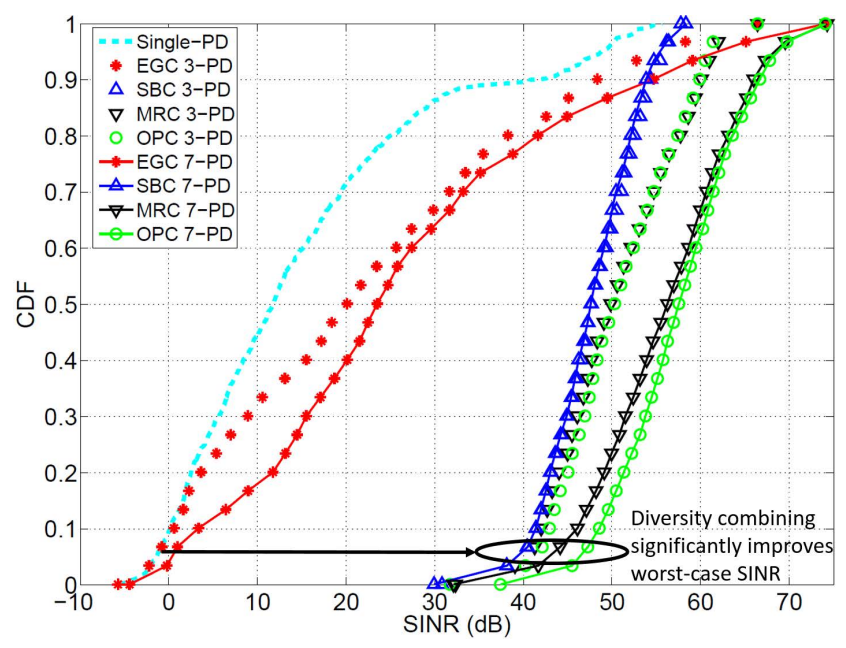

Figure 3: CDF of SINRs for different combining schemes for the single-LED AP configuration in Fig. 1(b). Receiver parameters: Single PD $\beta_{\mathrm{FOV}}=35^{\circ}, 3 \mathrm{PD}$ and $7 \mathrm{PD} \beta_{\mathrm{FOV}}=22^{\circ}$ and $\beta=15^{\circ}$.

is not possible due to physical limitations of piezoelectric actuators, a handover may be considered either to another LED in the same AP, or to a different AP.

\section{SIMULATION RESULTS}

In this section, we investigate the SINR distribution with simulations for different transmitter and receiver configurations and deployment scenarios. A room with dimensions of $19.5 \mathrm{~m} \times 10.4 \mathrm{~m} \times 4 \mathrm{~m}$ is considered. The deployment scenarios of the VLC transmitters are illustrated in Fig. 1(b) and Fig. 1(c). In order to achieve a fair comparison between different deployment scenarios, in total, there are 40 LEDs deployed in the single-LED transmitter scenario, and 42 LEDs deployed for the 7-LED transmitter scenario. Due to the geometrical constraints, we did not use the exactly same number of LEDs in these two scenarios. However, the impact of this difference is negligible in the final results.

The tilt angle $\alpha$ for the 7-LED transmitter is set to $45^{\circ}$, which is chosen heuristically after a wide simulation campaign to give maximum overall SINR [5]. Other simulation parameters are given in Table 1. By changing location of the receiver, the empirical CDF of SINR is obtained in the room. The height of the receiver is always set to $0.85 \mathrm{~m}$, and the receiver is always assumed to be looking upwards. Up to four multipath reflections are considered in all simulations.

\subsection{SINR CDFs For Different Configurations}

In Fig. 3, CDF of SINR for different receiver types and combining schemes are illustrated considering single LED

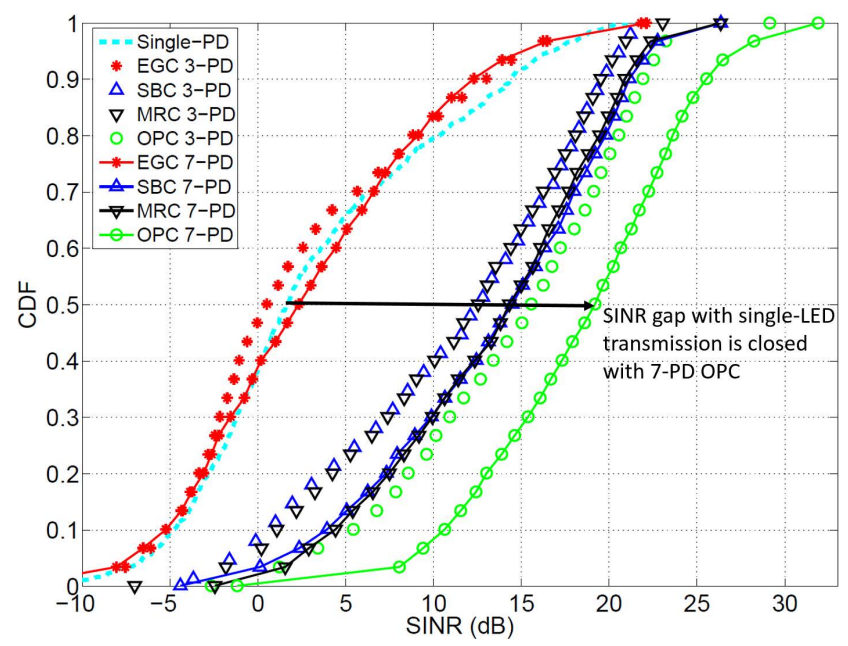

Figure 4: CDF of SINRs for different combining schemes. As in Fig. 1(c), 7-LED AP configuration is used. Receiver parameters: Single PD $\beta_{\mathrm{FOV}}=55^{\circ}, 3 \mathrm{PD}$ and $7 \mathrm{PD} \beta_{\mathrm{FOV}}=38^{\circ}, \beta=30^{\circ}$.

transmitter configuration. Single-PD receiver yields an SINR between $-5 \mathrm{~dB}$ and $55 \mathrm{~dB}$, while 3 -PD receiver yields an SINR between $35 \mathrm{~dB}$ and $65 \mathrm{~dB}$ except when EGC is used. For 7-PD case, MRC and OPC yields SINR between $35 \mathrm{~dB}$ and $75 \mathrm{~dB}$, and outperform 3-PD receiver. In general, MRC performs better than SBC, and OPC performs slightly better than MRC. Since OPC uses the correlation information at reflections from walls, it provides better performance. However, since the dominant component of the signal is LOS for the single-LED transmitter configuration, there is no significant difference between MRC and OPC.

Fig. 4 is similar to Fig. 3, except that, 7-LED transmitter configuration in Fig. 1(c) is used. In this configuration, since the LEDs are closer to each other at multi-element transmitters, they are not placed evenly in the room, and they cause significant interference to each other. Therefore, these transmitters are disadvantaged in terms of SINR performance, which introduces an SINR gap with the singleLED configuration in Fig. 1(b). However, as mentioned before, multi-element transmitters are still preferable due to low cost, and ease of installation and maintenance. In this scenario of Fig. 4, OPC provides much higher gain than MRC. For example, in 7-PD receiver case, OPC gives $5 \mathrm{~dB}$ 50 th percentile SINR gain over MRC and SBC. The reason for that is, LEDs are directed towards different angles as shown in Fig. 1(c), and wall reflection plays more important role in the overall received signal. This makes OPC a much better option when angle diversity transmitters are used. We observe that MRC shows nearly same performance with SBC. This behavior can be explained by the fact that, due to the large tilt angle between PDs, they look at different directions and do not receive direct signals from the same LEDs. Therefore use of data from other PDs does not improve SINR in this case.

In general, we observe that while single element receiver yields $-10 \mathrm{~dB}$ to $20 \mathrm{~dB}$ SINR in this configuration, 3-PD OPC receiver provides a $13 \mathrm{~dB} 50$ th percentile SINR gain, whereas 7-PD receiver provides a $17 \mathrm{~dB} 50$ th percentile SINR gain. Therefore, we can say that multi-element receivers significantly alleviate the low SINR problem of multi-element transmitters. 


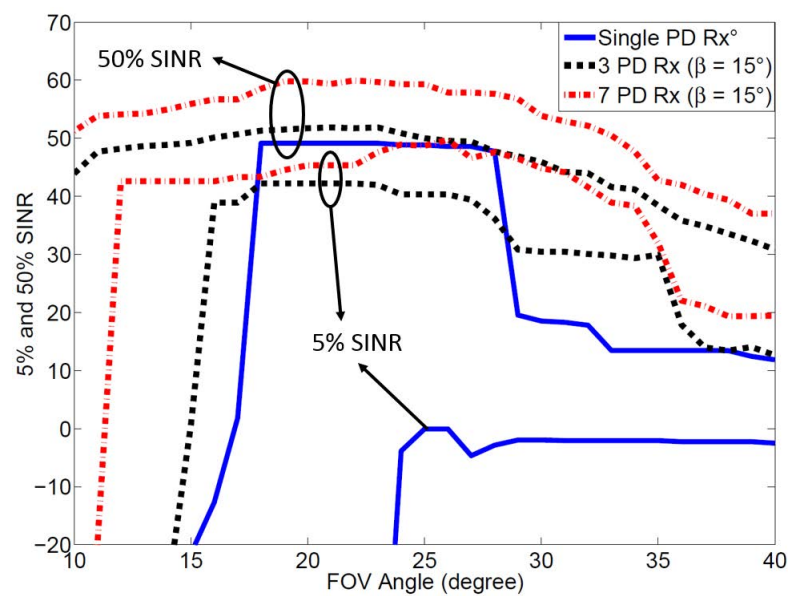

(a) $5 \%$ and $50 \%$ SINRs with different FOVs (Single-LED Tx).

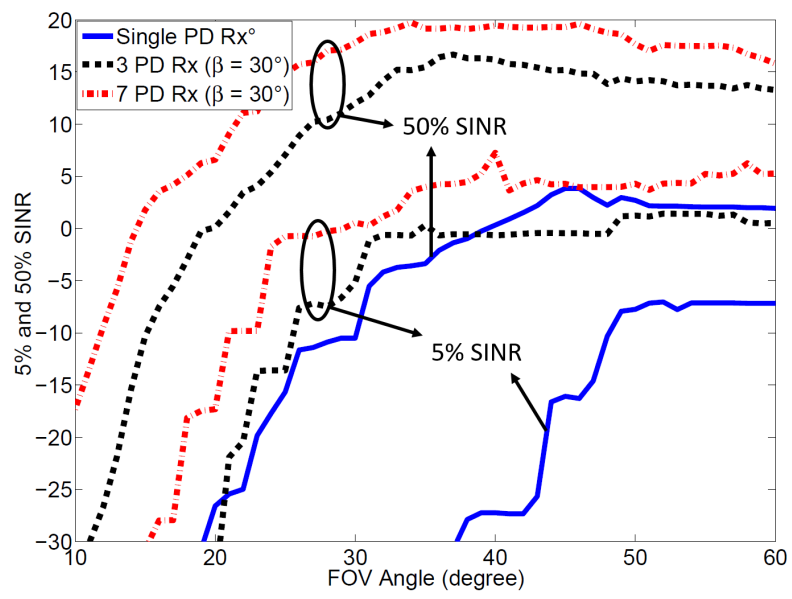

(c) $5 \%$ and $50 \%$ SINRs with different FOVs (7-LED Tx).

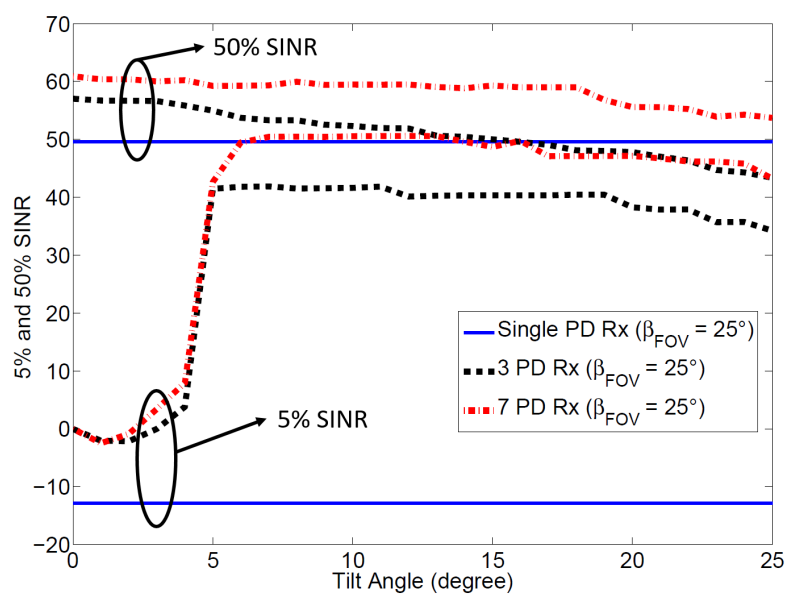

(b) $5 \%$ and $50 \%$ SINRs vs different tilt angles (Single-LED Tx).

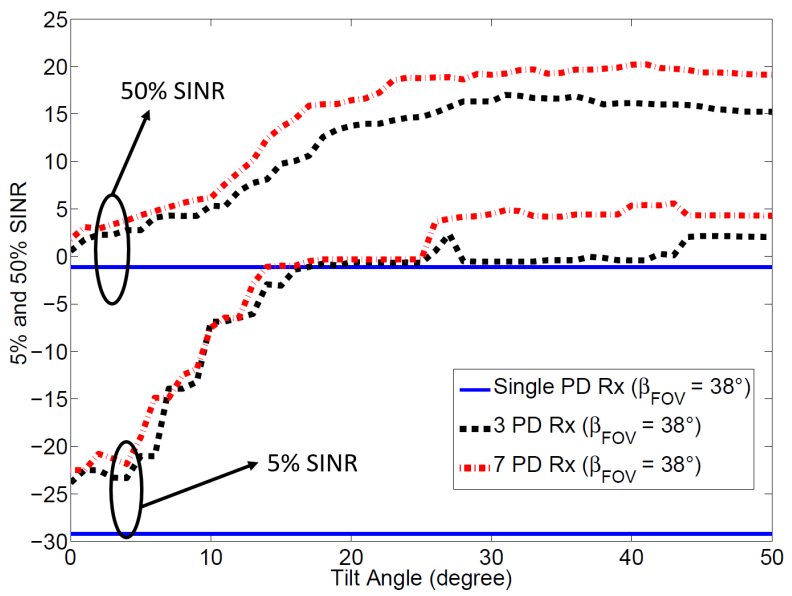

(d) $5 \%$ and $50 \%$ SINRs with different tilt angles (7-LED Tx).

Figure 5: $5 \%$ and 50\% percentile SINRs with different configurations.

\section{$5.25 \%$ and $50 \%$ SINR Performance}

In Fig. 5, 5\% and 50\% SINR performances of different transmitter and receiver configuration pairs are illustrated. $5 \%$ SINR means the lowest $5 \%$ value among all SINR values in the room. While $5 \%$ SINR captures the worst-case SINRs, $50 \%$ gives the median of the SINR distribution. In all subfigures, a blue solid line stands for single-PD receiver, black dashed line for 3-PD receiver, and red dot-dashed line for 7PD receiver. There are two lines for each; higher one always represents the $50 \%$ SINR, while the lower one represents the $5 \%$ SINR. Optimal combining is used for all scenarios at multi-element receivers, as it maximizes SINR in all cases.

In Fig. 5(a) and Fig. 5(b), single-LED transmitter configuration shown in Fig. 1(b) is used. Fig. 5(a) compares performances of receivers for different FOVs $\left(\beta_{\mathrm{FOV}}\right)$ of the PDs. At single-PD receiver, to maximize $50 \%$ SINR, FOV between 18 to 28 degrees can be used. On the other hand, FOV below 24 degrees yields unacceptable $5 \%$ SINRs, and therefore using a FOV between 24 degrees to 28 degrees will simultaneously yield good $50 \%$ and $5 \%$ SINRs for single LED transmitter case. Likewise, to maximize $5 \%$ and $50 \%$ SINRs jointly, 18 to 23 degrees of FOV can be used for 3-PD receiver, and 24 to 26 degrees can be used for 7-PD receiver.

For a single-LED transmitter scenario, Fig. 5(b) compares
$5 \%$ and $50 \%$ SINR performances for different $\beta$, which is the tilt angle between LEDs and the normal of multi-element receiver. In this figure, the performance of single element receiver is constant since it does not have a tilt angle. When the tilt angle of multi-element receivers is set to zero, all the PDs will look at the same direction. Therefore there is no significant gain with respect to single-PD receiver. There is only a limited gain because of increasing receiver surface area. While 6 to 15 degrees are best for 7-PD receiver, 5 to 10 degrees are optimal for 3-PD receiver.

Fig. 5(c) and Fig. 5(d) compares performance for different FOV and tilt angles for the 7-LED multi-element transmitter configuration in Fig. 1(c). In single LED case, light was always vertically transmitted from the ceiling, and there was an AP at each 1.5 meters. This makes a narrow FOV and small tilt angle the best option, since it receives the most signal from the desired LED, and does not receive much interference from the neighboring LEDs. However, in 7 LED transmitter case, it is a small probability for the receiver to be right under the AP, since the APs are sparsely placed. Because of the tilt of LEDs, the light comes with different angles in most regions. Therefore, in order to capture sufficient light intensity, optimal FOV and tilt angle are much larger than the previous case. 


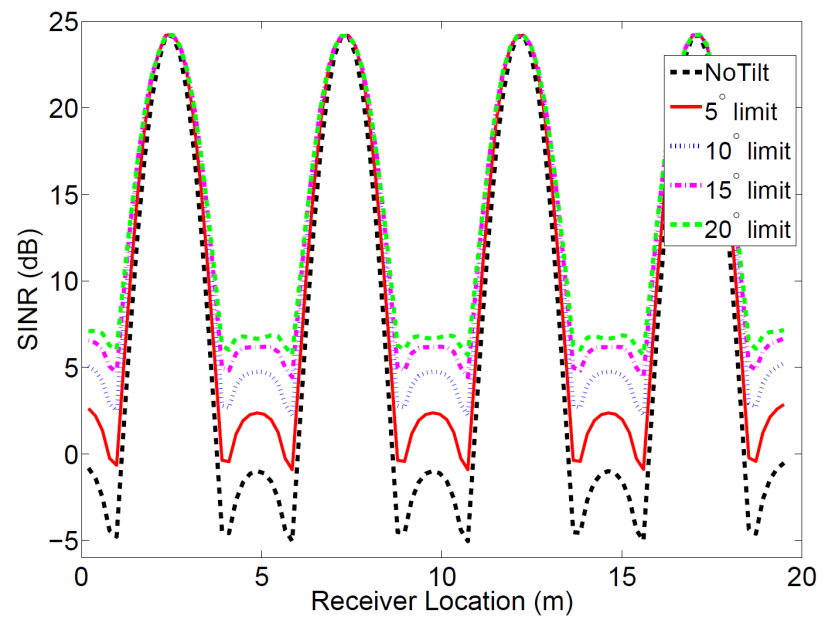

Figure 6: SINR for beam steering scenario considering different tilting angles and different user locations as in Fig. 2(b).

In order for the 7-PD receiver to give good SINR in the 7LED transmitter scenario, FOV should be 35 to 48 degrees. On the other hand, for the 3-PD receiver, 35 to 58 degrees are good. For single-PD receiver, 50 to 60 degrees give the best SINR. As seen in Fig. 5(d), for the 7-LED transmitter scenario, LED tilt around 30 degrees provides peak SINRs for both multi-element receivers.

\subsection{Effect of Beam Steering on SINR}

In Fig. 6, SINR distribution on the path illustrated in Fig. 2(b) is shown. Due to limited space, only single-LED transmitter and single-PD receiver configuration is presented. We made different simulations based on assumed steering ability of the LEDs. In Fig. 6, the locations with high SINR corresponds to regions beneath LEDs where no steering is required. In low SINR regions, which corresponds to cell edges, gain caused by beam steering is more visible. Without steering, the SINR drops to $-5 \mathrm{~dB}$ levels. When maximum 5 degree steering is applied, the SINR is always higher than $-1 \mathrm{~dB}$, which means a $4 \mathrm{~dB}$ gain. When the steering limit is increased to 10 degree, SINR gain is $7 \mathrm{~dB}$ at lowest SINR region. When more steering is applied, there is diminishing returns in SINR gains. In Fig. 7, the CDF of SINR throughout the room in Fig. 1(b) is illustrated. Likewise, the SINR is improved in all low and medium SINR regions.

The most important advantage of beam steering in VLC is that, it improves link quality especially in low SINR regions and cell edges. With beam steering, SINR level difference between low and high SINR regions is less, which is important for seamless communication. It is also important to have a good SINR at cell edges, since it will help for a softer handover between VLC APs. A disadvantage of beam steering is the cost of piezoelectric actuators and driving circuit, which will increase with higher steering ability. Evaluating trade off between cost and merits is left as a future study.

\section{CONCLUSION}

In this paper, SINR performances of multi-element VLC transmitters and receivers are jointly investigated, with different diversity combining techniques at multi-element receivers. As expected, OPC is the best receiver in all scenarios, while it provides higher gains over single PD scenario

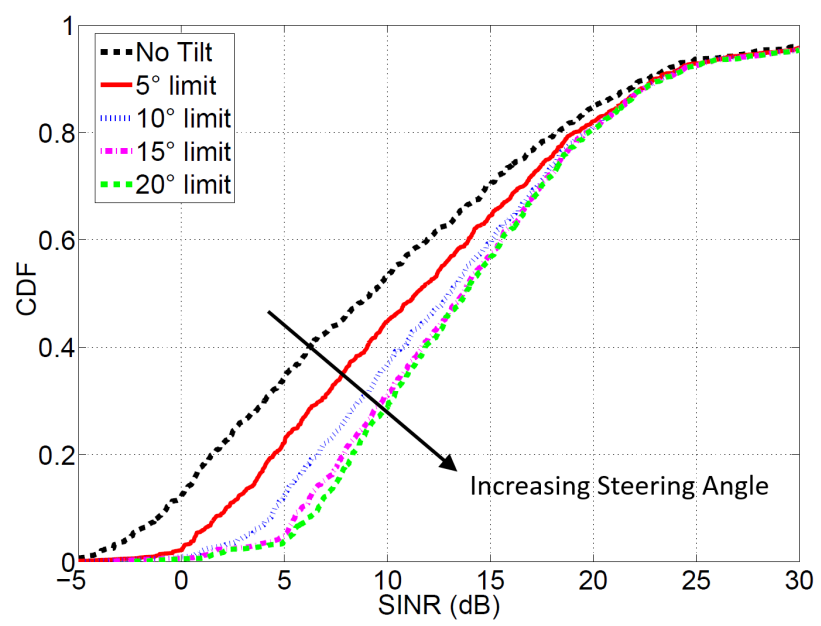

Figure 7: CDF of SINR for beam steering scenario.

when multi-element transmitters are used. With the use of multi-element transmitters, optimal value of FOV and tilt angle increases at the receiver. Effect of VLC beam steering on SINR is also studied, which is shown to provide up to $12 \mathrm{~dB}$ SINR gain in the low SINR region. Our future work includes assigning LEDs to users optimally in multi user scenarios.

\section{REFERENCES}

[1] Y. S. Eroglu, I. Guvenc, N. Pala, and M. Yuksel, "AOA-based localization and tracking in multi-element VLC systems," in Proc. IEEE Wireless and Microwave Tech. Conf. (WAMICON), Cocoa Beach, FL, Apr. 2015.

[2] A. Şahin, Y. S. Eroğlu, I. Guvenc, N. Pala, and M. Yüksel, "Hybrid 3-D localization for visible light communication systems," IEEE J. Lightwave Technol., vol. 33, no. 22, pp. 4589-4599, Nov. 2015.

[3] M. F. Keskin and S. Gezici, "Comparative theoretical analysis of distance estimation in visible light positioning systems," IEEE J. Lightwave Technol., vol. 34, pp. 854-865, Feb. 2016.

[4] Y. S. Eroglu, A. Sahin, I. Guvenc, N. Pala, and M. Yuksel, "Multi-element transmitter design and performance evaluation for visible light communication," in Proc. IEEE GLOBECOM Wkshp., San Diego, CA, Dec. 2015.

[5] P. Palathingal, I. Guvenc, N. Pala, and M. Yuksel, "A multi-element vlc architecture for high spatial reuse," in Proc. ACM Workshop on VLCS, 2015.

[6] L. Yin, X. Wu, and H. Haas, "Indoor visible light positioning with angle diversity transmitter," in Proc. IEEE Vehic. Technol. Conf. (VTC), Boston, MA, Sep. 2015.

[7] C. Gong and Z. Xu, "Linear receivers for optical wireless scattering communication with multiple photon detectors," in Proc. IEEE GLOBECOM Wkshp., Austin, TX, 2014.

[8] A. Nuwanpriya, S.-W. Ho, and C. S. Chen, "Angle diversity receiver for indoor MIMO visible light communications," in Proc. IEEE GLOBECOM Wkshp., Austin, TX, Dec. 2014.

[9] A. Tsiatmas, F. Willems, and S. Baggen, "Optimum diversity combining techniques for visible light communication systems," in Proc. IEEE GLOBECOM Wkshp., Austin, TX, Dec. 2014.

[10] Z. Chen, D. Tsonev, and H. Haas, "Improving SINR in indoor cellular visible light communication networks," in Proc. IEEE Int. Conf. Commun. (ICC), Sydney, Australia, June 2014.

[11] F. Claeyssen, R. Le Letty, F. Barillot, N. Lhermet, and H. Fabbro, "Mechanisms based on piezoactuators," in Proc. SPIE, vol. 4332, 2001.

[12] J. Barry, J. Kahn, W. Krause, E. Lee, and D. Messerschmitt, "Simulation of multipath impulse response for indoor wireless optical channels," IEEE J. Select. Areas Commun. (JSAC), vol. 11, no. 3, Apr. 1993.

[13] J. Winters, "Optimum combining in digital mobile radio with cochannel interference," IEEE J. Select. Areas Commun. (JSAC), vol. 2, no. 4, pp. 528-539, July 1984. 